\title{
Polymeric Proteins, Rheological Properties andBREADMaking Quality of Durum Wheat Flours and Semolina
}

\author{
Abdenour Yesli $^{1}$, Ghania Ounane ${ }^{2}$ and Chahinez Tazrout ${ }^{2}$ \\ Faculté des Sciences Biologiques et des Sciences Agronomiques. Université Mouloud Mammeri, Tizi Ouzou. \\ 2 Département de Technologie des IAA, Ecole Nationale Supérieure des Sciences Agronomiques, Hassan Badi, \\ 16200 Alger, Algérie
}

\begin{abstract}
Algerian commercial durum wheat flours and semolina from different millers was evaluated for flours/ Semolina mixed properties, dough physical characteristics and baking quality. Variation of protein fractions was studied. Durum flours and semolina exhibited higher glutenin concentration, medium SDS unextractable polymeric proteins. All samples used in this study had higher starch damage and falling number value Starch damage affects negatively alveograph data (tenacity/extensibility ratio and deformation energy). Farinograph and mixograph indicated both flours and semolina rated best for bread making. Morphological image analysis was applied to characterise each bread crumb type. Bread made with flours characterised by smaller cells, thinner walls and finer crumb
\end{abstract}

Keywords: Durum flour, Durum semolina, Polymeric proteins, Alveograph, Farinograph, Mixograph, Image analysis.

\section{Introduction}

Durum wheat is an important crop of Algerian diet used to produce several common foodstuffs such as couscous, pasta, frik and various types of traditional flat bread. Protein quality and quantity are considered primary factors in measuring the potential of flour/semolina in relation to end use. Stored proteins determine dough functional properties. In addition, quality is still heavily dependent on sevral other tests such as alveograph used to predict baking quality. It measures the resistance to biaxial extension obtained from a thin sheet of flour water- salt. The farinograph measures and records resistance of dough to mixing. It is used to evaluate water absorption and to determine stability and other characteristics of doughs during mixing. The mixograph measures the power used to mix the dough or the resistance to the dough. Baking is the final test and indicates the quality of final product. In this paper, proteins quantity and quality of durum wheat flours and semolina were investigated. Rheological and properties of products were assessed. Additionaly, breads were produced using standard method of bread making.

\section{Materials and Methods}

\subsection{Samples}

Twelve samples were used. Samples of commercial flour and semolina supplied from three industrial Millers with the same diagram of milling were durum flour (DS), durum first clear flour (DD1), durum first clear flour 4 (DD4), and Durum semolina (DS).

\subsection{Physico-chemical properties}

Protein content (\%N X 5.7) was determined by the Kjeldahl method according to AACC approved method (46-19.01), Wet gluten content (\%) is determined by washing the dough obtained from wheat flour/semolina, with $2 \% \mathrm{NaCl}$ solution to remove the starch and other soluble compounds of the sample. Gluten index were determined using Glutomatic perten instruments (AB type 2200, Huddinge, Sweden) according to AACC standard method (38-12.02). Sodium dodecyl sulfate (SDS) sedimentation test for assessed the gluten strength. The SDS - sedimentation volume of wheat was determined by a modified method as described [1]. Starch Damage was determined using the kit Megazyme Starch Damage (Megazyme Int., Wicklow, Ireland). Falling 
number was determined according to ICC standard method using Falling Number 1500 (Perten Instruments AB, Sweden).

\subsection{Proteins analysis}

Determination of polymeric protein, gliadin, albumins + globulins $(A+G)$ and SDS - unextractable polymeric proteins (UPP) carried out using respectively the method [2][3] and [4].

\subsection{Rheological analysis}

Alveogrpah properties of the flours/semolina were obtained using the method of the Chopin- sa, Villeneuvela-Garenne - France. The resulting alveograms were used to detrmine the over - pressure $(\mathrm{P}, \mathrm{mm})$ as an indicator of dough tenacity or resistance to deformation, the deformation energy $\left(\mathrm{W}, 10^{-4}\right)$ required to inflate the doughbubble until it ruptures. The configuration ratio $\mathrm{P} / \mathrm{L}$, an indicator of the rheological balance of the dough. Farinograph properties of the flours/semolina were obtaiened using the standard ICC Brabender Farinograph method ( 115/1 ). The farinograph was used to determine dough development time (Far DDT), farinograph stability (Far STAB), and mixing tolerance index (MTI). A complementary method of measuring dough mixing properties was applied using a mixographic. Parameters were determined in a $30 \mathrm{~g}$ Mixograph according to the AACC method 54-40A .The mixing parameters measured were Peak dough development (MTP, min), peak dough resistance (PDR, \%).

\subsection{Baking procedure}

The Formulation, based BIPEA information, included flour/semolina $(100 \%)$, water (farinograph $+9 \%$ ), salt $(2.2 \%)$, yeast $(2.5 \%)$.

\subsection{Image analysis}

For image analysis leavend breads produced in durum flours and semolina. Easch loaf of bread was sliced in regular slices to characterize cell size with erosion- dilatation method [5].

\section{Results and Discussion}

\subsection{Physico-chemical properties.}

Characteristics of Algerian commercial durum flours and Semolina are shown in table I. The highest protein content was observed in DFCF4 which showed also the smallest gluten and, was poorly extensible, with a gluten index value 39 and medium sediment volume in sodium dodecyl sulphate, while Durum semolina was characterised by the lowest values of both proteins content and volume sediment. Results showed all durum flours and semolina had the highest value of falling number 478 to $567 \mathrm{~s}$. The higher damage starch $8.13 \%$ and $7.12 \%$ were observed in DFCF4 and DF, respectively. In agreement [6], milling treatement had a large effect upon starch damage values.

TABLE I. Flours and semolina physico-chemical properties

\begin{tabular}{lcccc}
\hline Paramètres & DFCF1 & DFCF4 & DF & DS \\
\hline Protéins $(\% \mathrm{dw})$ & $10.83 \pm 0.57$ & $13,44 \pm 0,40$ & $11,37 \pm 0.67$ & $11,34 \pm 0.13$ \\
Wet Gluten $(\%)$ & $22.92 \pm 3.07$ & $24.45 \pm 3.66$ & $27.33 \pm 3.53$ & $26.54 \pm 1.31$ \\
Gluten index $(\% \mathrm{~ms})$ & $58 \pm 8$ & $39 \pm 4$ & $48 \pm 9$ & $56 \pm 7$ \\
SDS (ml) & $50 \pm 4$ & $45 \pm 6$ & $45 \pm 7$ & $31 \pm 3$ \\
FNS (S) & $478 \pm 258$ & $511 \pm 199$ & $567 \pm 261$ & $556 \pm 227$ \\
Starch damage (AE \%) & $6.86 \pm 0.9$ & $8.13 \pm 0.94$ & $7.12 \pm 0.95$ & $5.93 \pm 2.37$ \\
\hline
\end{tabular}

\subsection{Variation of protein fractions}

The proportion of different fractions of protein of flour and semolina is illustrated in table II. Glutenin was the largest fraction ranging between 55,34 and 52,86\%, DFCF4 showed the highest and DFCF1 the lowest. Glutenin: Gliadin (Glu: Gli) ranged between 1.49 and 1.80, the highest ratio for DFCF4 and the lowest for DS were observed. A+B were present in the lower proportion ranging between 11,06 and 14,91\%. DFCF4 showed the highest proportion of A+ B among the samples studied while DS showed the lowest. UPP showed a wide variation among the flours / semolina with DFCF1 (43,3\%) having the lowest and DF (47,90\%) the highest 
proportion. The differences in extractability of the polymeric proteins could be attributed to differences in degree of polymerization (Singh et al, 2011).The difference of amount of UPP between samples can be explained from different quaternary structure [7].

TABLE II: Variation of protein fractions

\begin{tabular}{|c|c|c|c|c|c|}
\hline Parameters & GLU\% & GLI\% & GLU :GLI & $\mathrm{A}+\mathrm{B} \%$ & UPP\% \\
\hline DFCF1 & $52,86 \pm 3,51$ & $33,26 \pm 4,65$ & $1,63 \pm 0,29$ & $13,88 \pm 2,23$ & $43,3 \pm 2,64$ \\
\hline DFCF4 & $55,34 \pm 5,23$ & $29,76 \pm 3,76$ & $1,80 \pm 0,39$ & $14,91 \pm 1,48$ & $44,43 \pm 4.45$ \\
\hline $\mathrm{DF}$ & $53,58 \pm 2,5$ & $32,21 \pm 0,8$ & $1,67 \pm 0,07$ & $14,21 \pm 2,91$ & $47,90 \pm 3,03$ \\
\hline DS & $53,06 \pm 3,48$ & $35,88 \pm 0,90$ & $1,49 \pm 0,13$ & $11,06 \pm 2,70$ & $47,83 \pm 3,68$ \\
\hline
\end{tabular}

3.3 Rheological properties of flours/semolina

Table III give rheological data for flours and semolina. As for alveograph, P/L varied between flours and semolina. Tenacity was always higher than extensibility. Higher value of P/L was found in DFCF1. The high value of $\mathrm{P} / \mathrm{L}$ depend both milling process and climate. The stressed milling and high temperature effect tenacity[8]. The work corresponding to the deformation of the dough ranged from $118 \mathrm{X} 10^{-4} \mathrm{~J}$ in DFCF1 to 197 $\mathrm{X} 10^{-4} \mathrm{~J}$ in DS. The lower W of all samples can be attributed to higher value of $\mathrm{P}$. W values are more influenced by pressure.

Based on farinograph DDT and stability (STAB), durum flour first clear 4 showed a higher dough development time than other fractions. Differences in DDT and STAB can attributed to gluten strength [9] and milling conditions [10]. Differences in peak dough development between flours and semolina estimated by the mixograph can explained with differences in proteins content and the glutenin fraction of protein [11].

TABLE III: Rheological properties of flours and semolina

\begin{tabular}{|c|c|c|c|c|}
\hline Paramètres & DFCF1 & DFCF4 & DF & DS \\
\hline \multicolumn{5}{|c|}{ ALVEOGRAPH } \\
\hline $\mathrm{W}\left(10^{-4} \mathbf{J}\right)$ & $118 \pm 12$ & $114 \pm 35$ & $140 \pm 31$ & $197 \pm 29$ \\
\hline $\mathrm{P} / \mathrm{L}$ & $3.0 \pm 0.9$ & $3 \pm 0.9$ & $3.0 \pm 0.6$ & $4 \pm 0.45$ \\
\hline \multicolumn{5}{|c|}{ FARINOGRAPH } \\
\hline $\operatorname{DDT}(\min )$ & $2.4 \pm 0.7$ & $3.2 \pm 0.4$ & $2.2 \pm 1.1$ & $2.8 \pm 0.4$ \\
\hline \multicolumn{5}{|l|}{ MIXOGRAPH } \\
\hline $\operatorname{MTP}(\min )$ & $1.91 \pm 1.06$ & $1.30 \pm 0.15$ & $1.54 \pm 0.46$ & $2.66 \pm 0.59$ \\
\hline $\operatorname{PDR}(\%$ torque $)$ & $44.0 \pm 3.0$ & $43.0 \pm 8.0$ & $45.0 \pm 5.6$ & $48.0 \pm 4.7$ \\
\hline
\end{tabular}

\subsection{Crumb texture evaluation}

Crumb morphological for bread of different types are shown in Fig 1. These images reveal differences in the cell size distribution crumb. Erosion- dilation (ERDIL) curves indicates accurate view of these changes (fig 2). The bread made with flours can be clearly distinguished to semolina bread. The crumb structure of bread flours content smaller cells, thinner walls and finer crumb. 

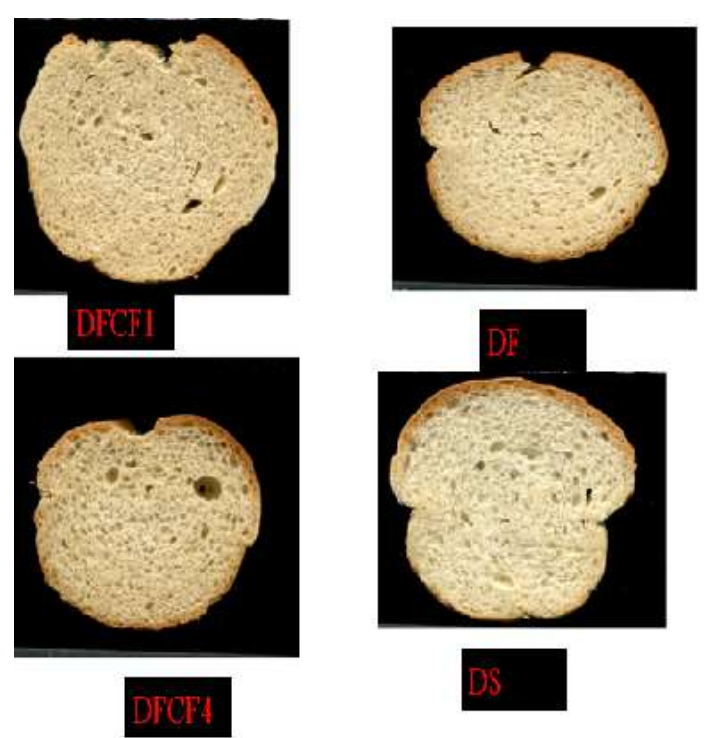

Fig. 1: Photographs of bread slices for different dough.

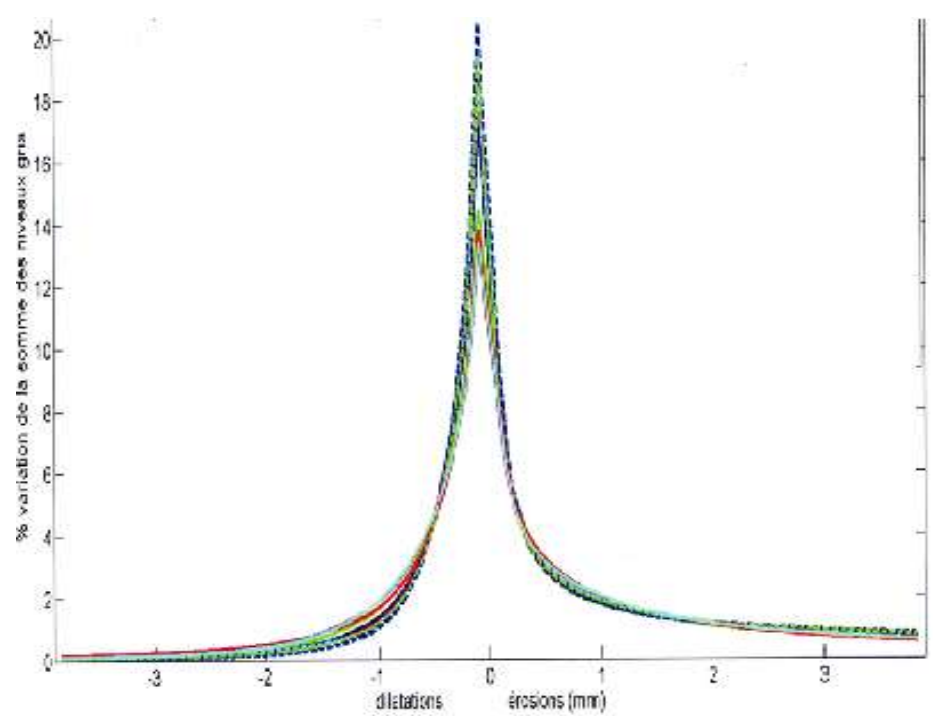

Fig. 2: Erosion- dilatation (ERDIL) curve for different dough.

\section{Conclusion}

The variations of protein fractions, rheological and bread making quality of Algerian durum wheat fractions (DFCF1, DFCF4, DF and DS) were studied. Breads of good qualities were obtained. The tests showed differences between different fractions. The general profile of alveograph curves of flour and semolina is generally revealed reduced dough extensibility and increased tenacity. The mixograph test and farinograph showed good baking quality of all fractions tested. Image analysis revealed the flours breads are finer crumb, small cells than semolina breads.

\section{References}

[1] J. E. Dexter, R. R. Matsuo, F. G. Kosmolak. D. Leisle, and B. A .Marchylo,. (1980). The suitability of the SDSsedimentation test for assessing gluten strength in durum wheat. Canadian Journal of Plant Science, 60(1), 25-29. http://dx.doi.org/10.4141/cjps80-004

[2] J. William, Hurkman, and K. Tanaka .Charlene. 2004."Improved methods for separation of wheat endosperm proteins and analysis by two-dimensional gel electrophoresis." Journal of cereal science 40.3: 295-299. http://dx.doi.org/10.1016/j.jcs.2004.09.001

[3] N.K.Singh, K. W. Shepherd, and G. B. Cornish.1991. "A simplified SDS-PAGE procedure for separating LMW subunits of glutenin." Journal of Cereal Science 14.3 : 203-208.

http://dx.doi.org/10.1016/S0733-5210(09)80039-8

[4] R. B .Gupta, K. Khan, and F Macritchie.1993. "Biochemical basis of flour properties in bread wheats. I. Effects of variation in the quantity and size distribution of polymeric protein." Journal of Cereal Science 18.1 : 23-41.

http://dx.doi.org/10.1006/jcrs.1993.1031

[5] J.Rouillé,J.M. Bonny, G. Della Valle, M.F.Devaux, and J.P. Renou. 2005. Effect of flour minor components on bubble growth in bread dough during proofing assessed by magnetic resonance imaging. Journal of agricultural and food chemistry, 53(10), 3986-3994.

http://dx.doi.org/10.1021/jf047953r

[6] K. R. Preston, R. H. Kilborn, and J. E. Dexter.1987. "Effects of starch damage and water absorption on the alveograph properties of Canadian hard red spring wheats." Canadian Institute of Food Science and Technology Journal 20.2: 7580.

http://dx.doi.org/10.1016/S0315-5463(87)71093-1 
[7] E. Lemelin, T. Aussenac, L. Salvo,and V. Lein. 2004. The impact of variety and environment on the size characterizatics of wheat proteins using asymmetrical flow field-flow fractionation and multi-angle laser light scattering. Cereal Chem. 82: 28-33.

http://dx.doi.org/10.1094/CC-82-0028

[8] B. Borghi, R. Castagna, M. Corbellini, M. Heun, and F. Salamini.1996. Breadmaking quality of einkorn wheat (Titicum monococcum ssp.monococcum). Cereal Chem. 73:208-214.

[9] K. Ammar, W.E. Kronstad, and C.F. Morris. 2000. Bread- making quality of selected durum wheat genotypes and relationship with high molecular weight glutenin subunits allelic variation and gluten protein polymeric composition. Cereal Chemistry. 77: 230-236.

http://dx.doi.org/10.1094/CCHEM.2000.77.2.230

[10] E.S. El-Porai , A.E. Salama, A.M. Sharaf, A.I. Hegazy, and M.G.E. Gadallah. 2013. Effect of different milling processes on Egyptian wheat flour properties and pan bread quality. Annals of Agricultural Science. 58(1), 51-59. http://dx.doi.org/10.1016/j.aoas.2013.01.008

[11] V. K. Rao, S. J. Mulvaney, J. E. Dexter, N. M. Edwards and D. Peressini. 2001. Stress-Relaxation Properties of Mixograph Semolina-Water Doughs from Durum Wheat Cultivars of Variable Strength in Relation to Mixing Characteristics, Bread- and Pasta-making Performance. Journal of Cereal Science 34 (2001) 215-232

http://dx.doi.org/10.1006/jcrs.2001.0384 